\title{
Secondary System I nitialization Protocol Using FFT-based Correlation Matching for Cognitive Radio Ad-hoc Networks
}

\author{
Sang-Jo Yoo ${ }^{1}$, Ju-Tae Jang ${ }^{1}$, Myunghwan Seo ${ }^{2}$, Hyung-Weon Cho ${ }^{2}$ \\ $\mathbf{1}_{\text {School of Information and Communication Engineering, Inha University }}$ \\ 253 Yonghyun-dong, Nam-gu, Incheon 402-751, Korea \\ $\mathbf{2}$ TICN R\&D Center Hanwha Thales Co., Ltd, Korea \\ [e-mail: sjyoo@inha.ac.kr] \\ *Corresponding author: Sang-Jo Yoo
}

Received May 29, 2016; revised September 12, 2016; accepted October 17, 2016; published January 31, 2017

\begin{abstract}
Due to the increasing demand for spectrum resources, cognitive radio networks and dynamic spectrum access draw a lot of research into efficiently utilizing limited spectrum resources. To set up cluster-based CR ad-hoc common channels, conventional methods require a relatively long time to successfully exchange the initialization messages. In this paper, we propose a fast and reliable common channel initialization protocol for CR ad-hoc networks. In the proposed method, the cluster head sequentially broadcasts a system activation signal through its available channels with a predetermined correlation pattern. To detect the cluster head's broadcasting channels and to join the cluster, each member node implements fast Fourier transform (FFT) and computes autocorrelation of an FFT bin sequence for each available channel of the member node. This is compared to the predetermined reference pattern. The join request and channel decision procedures are also presented in this paper. In a simulation study, the performance of the proposed method is evaluated.
\end{abstract}

Keywords: cognitive radio network, common channel, CR ad-hoc, secondary system initialization

A preliminary version of this paper appeared in ICUFN 2015, Sapporo, Japan, July 2015 [13]. This version includes a concrete analysis, protocol details and performance evaluations for various wireless cahnnel conditions. This work was supported by a grant-in-aid of HANHWA THALES. 


\section{Introduction}

$\mathbf{W}_{\text {ith the rapid deployment of new wireless devices and applications, the demand for }}$ wireless radio spectrum is also increasing. Therefore, wireless radio spectrum has become a scarce commodity in many developed and developing countries. According to the Federal Communications Commission (FCC), the United States will run out of radio spectrum in the upcoming years. The spectrum regulatory bodies in different countries assign spectrum to licensed users, also termed primary users (PUs), on a long-term persistent basis for large geographical regions. However, a large portion of the assigned spectrum remains underutilized [1]. Therefore, in order to improve utilization of the spectrum, with an aim to address the critical problem of spectrum scarcity, the possibility of opening licensed frequency bands to unlicensed operations has been considered [2-4]. Generally, licensed users or PUs have the right to utilize the spectrum without interference. However, unlicensed users, also known as secondary users (SUs), are allowed to opportunistically exploit unused licensed spectrum, called spectrum holes, to send data. If a PU is currently not using the spectrum, the SU changes its transmission parameters to take advantage of the spectrum hole. After discovering the unoccupied frequency bands, an SU selects the best available channel for opportunistic communications. The SU must vacate the channel when the PU returns.

Cognitive radio (CR) devices equipped with a dynamic spectrum access capability are able to detect spectrum opportunities in licensed bands and map them into logical channels, which can be temporarily used by the CR nodes (secondary users) for communications. Therefore, CR is regarded as a promising technology to cope with the spectrum scarcity problem. In CR networks, unlicensed users are allowed to use temporarily unused licensed bands, as long as they do not interfere with the communications of licensed users [5]. One of the key concerns in using CR technology is to guarantee that PU transmissions are always secured. To achieve this, it is vital to exchange information pertaining to the spectrum availability between a given node pair before starting data transmission.

The methods to successfully negotiate common channels among SUs have been taken into consideration [6], and can be classified into sequence-based methods and group-based methods. In a sequence-based method, each node changes its negotiating channel in accordance with the random or predetermined channel-hopping sequence [7]. For example, DaSilva et al. [8] used permutations of all the available channels to construct sequences. The sequence-based design may require significant time to re-establish a new control channel. Also, link-by-link connection establishment incurs high control overhead. In a group-based method, SUs in proximity to each other negotiate using the channels commonly available to them [9]. As SUs in this method adaptively update their choice of channels, the common channel allocation may fluctuate with the updates of neighbors' choices, and hence, the performance of this method may not be consistent. Kim and Yoo [10] proposed a multicasting tree-based initial common channel setup method for multi-hop ad-hoc networks. A method to reduce rendezvous time with a hop-and-wait channel hopping method was also presented by Chuang et al. [11]. In [12], as a group-based rendezvous protocol a cyclic listening method was proposed.

CR networks can either be centralized or distributed. In a centralized CR network, a central entity controls spectrum allocation and spectrum access, and also gathers information about the radio environment. In distributed CR networks, there is no central unit to gather information about the radio environment. Heterogeneous spectrum availability and the 
absence of a central control entity in CR ad-hoc networks demand self-organization of CR users for resourceful spectrum coordination. Cluster-based distributed ad-hoc CR networks organize nodes into clusters in order to provide network-wide performance enhancement. There are three main advantages brought about by clustering in CR networks, namely stability, scalability, and supporting cooperative tasks, such as channel sensing and channel access, which are essential to CR operations, and these advantages have led to the use of clustering in CR networks. The cluster-based ad-hoc cognitive radio networks can be applied to many application scenarios such as military ad-hoc networks, campus networks, vehicular ad-hoc networks and disaster safety communication networks.

In a CR wireless network, due to location-dependent channel statistics, the available channel set for each secondary member node can be different from other nodes. Especially, in military CR environments, the distance between member nodes can range from a hundred meters to several kilometers, and as a result, the channel list available to each member node can be different. From the different available channels between member nodes, common available channel (or channels) should be selected for the cluster-based ad-hoc network. The common available channel can be used for data and control message transmission between the member nodes. In the typical communication protocols of CR ad-hoc networks, the existence of a dedicated common control channel for control message is assumed to exchange each member node's available channel list. In this case, cluster coordinator node simply decides one or multiple common available channels for data communication. However it shows weakness for the jamming attack on the dedicated control channel and the control traffic can be also saturated. There, in this paper we assume that there is no predetermined control channel for the CR cluster so that we need an initialization protocol that helps member nodes setup common data channel(s).

It is obvious that during the initialization phase, the joining of the member nodes in the cluster, the sensing information report, and final common data channel notification should be done quickly and reliably. Therefore, there must be an efficient mechanism for a CR-based transmitter and its intended receiver to pick a common available channel for transmission. If the channel negotiation mechanism is not designed appropriately, it may be unmanageable to achieve the proper utilization of resources and time overhead. In a cluster-based CR ad-hoc network, member nodes can reach a cluster head $(\mathrm{CH})$ directly, and cluster nodes need to share their sensing results and determine a common channel set. In the conventional group-based initialization protocol, the $\mathrm{CH}$ sequentially broadcasts a setup request message over all the CH's available channels, and each member node listens to one of its available channels during a predefined time. If a member node does not receive the setup request message, then it switches to its next available channel until it successfully receives the message. However, in the worst case scenario, the maximum delay may not be bound, or it requires a long setup time.

In this paper, we propose a novel, secondary, system initialization protocol for cluster-based CR networks. In the proposed method, the CH sequentially broadcasts a system activation signal on each available channel with a predetermined pattern. The system activation signal is a specific time domain waveform that has a pre-determined frequency-domain feature. Therefore by decoding the signal, member nodes of the cluster can easily know CH's network initialization request and the available channels of $\mathrm{CH}$. The activation signal sequence is designed to have a specific frequency-time correlation characteristic. To detect the cluster head's broadcast channels reliably and quickly, each node implements fast Fourier transform (FFT) at every defined time period and computes an autocorrelation for the FFT bin sequence for each channel available to the member node. The correlation is compared to the predetermined pattern. After finding out the CH's broadcast 
channel, the cluster-join procedure is followed. The common data channel set that is to be used for the cluster is determined by the $\mathrm{CH}$ and is broadcast to member nodes by the proposed sequential announcement method.

The rest of this paper is organized as follows. In Section II, the proposed secondary system activation mechanism is presented. In Section III, member node-join and CH common channel decision procedures are explained. In Section IV, the performance of the proposed method is evaluated, and finally, Section V concludes this paper.

\section{Secondary System Activation Mechanism}

\subsection{System Model}

Heterogeneous spectrum availability and the absence of a central control entity in CR ad-hoc networks necessitate self-organization of CR users for efficient spectrum coordination. The cluster-based organization in a CR ad-hoc network is known to be effective in reducing communications overhead and at providing efficient spectrum utilization. In this paper, we assume that there exists a cluster head and multiple member nodes within $\mathrm{CH}$ range. The $\mathrm{CH}$ initiates CR ad-hoc cluster formation and decides common channels for data transmission based on each member node's available channel report.

As shown in Fig. 1, in our model, the entire operational band is partitioned into $G$ band groups (BGs). Each BG consists of $\mathrm{M}$ channels, and each channel is subdivided into request and response sub-channels. The entire operational spectrum band, the channel bandwidth and the number of channels per band group are the operational parameters and they are pre-determined depending on the CR ad-hoc application scenarios by a system operator.

The request and response sub-channels are used for system activation and join replies by the $\mathrm{CH}$ and member nodes, respectively. For channel occupancy evaluation, spectrum sensing is performed in a channel-based way (not on sub-channels) by the $\mathrm{CH}$ and member nodes. In this paper, we assume that each member node performs local spectrum sensing and cooperative spectrum sensing is not considered.

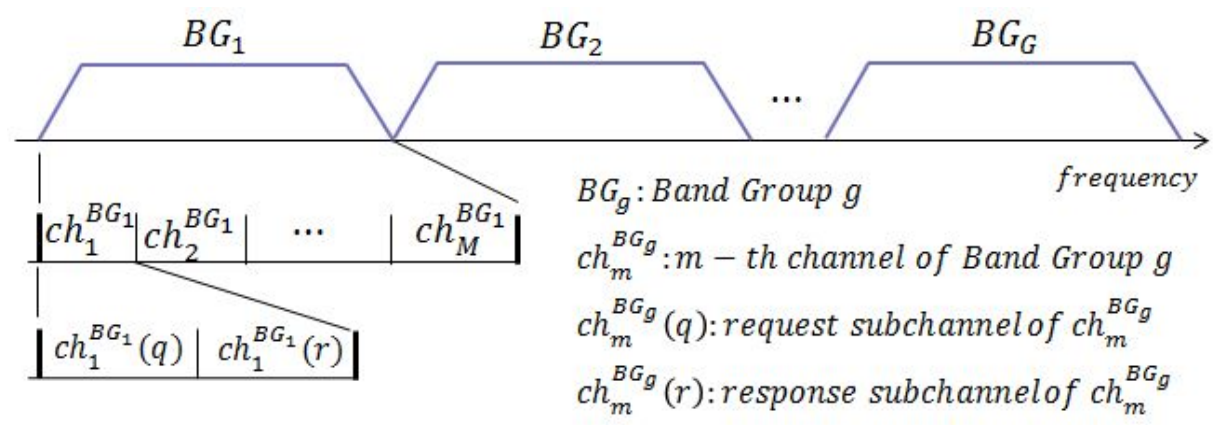

Fig. 1. Band group formation.

\subsection{System Activation by the Cluster Head}

Based on the local sensing results, the $\mathrm{CH}$ selects the best channels, $\boldsymbol{b}_{b g}$, up to max $b$ channels (predetermined) from among all available channels for all $B G s$. From all available channels, in which use of those channels do not give harmful interference to primary users, $\mathrm{CH}$ determines the best channels based on signal to interference plus noise ratio (SINR). The 
number of best channels for each BG is not fixed, and is an implementation issue. However, at least one channel has to be selected from a BG if the BG has available channels. Let $c h_{b(i) \in \boldsymbol{b}_{b g}}^{B G_{g}}$ be the $i$-th selected best channel of $B G_{g}, \forall g \in\{1,2 \cdots G\} . b_{b g}$ is the best channel set for band group $g$. Then, the $\mathrm{CH}$ sequentially broadcasts a system activation (SA) signal on the $c h_{b(i)}^{B G_{g}}(q)$ request sub-channel of all best channels.

It should be noted that the $\mathrm{CH}$ does not need to select best channels that equal max_b, but as in Fig. 2, the $\mathrm{CH}$ should follow the SA transmission circulation cycle $T_{c}=\left(T_{s} \times 2\right) \times$ $\max \_b$ to make the predefined correlation feature. $T_{s}$ is the sub-channel time, and the $\mathrm{CH}$ stays on the request and response sub-channels to send the SA signal and to receive join messages from member nodes during $T_{s}$. After $T_{c}$ time, the $\mathrm{CH}$ returns to the first selected channel and repeats the system activation behavior until the repeated cycles reach the predefined maximum number of SA transmission cycles (max_cycle).

The SA signal is not a message, but it can be any band-limited signal waveform. The only required condition is that the transmission power of the SA signal should be constant during the system activation period. Fig. 2 shows the system activation signal transmission on the best channels of the band groups. As shown in Fig. 2, the SA signal, represented by solid square box, is sequentially broadcast by the $\mathrm{CH}$ on the request sub-channels of the best channels for each $B G$. When the $\mathrm{CH}$ reaches the last best channel of all band groups, it returns to the first best channel and repeats the SA broadcast. If the number of best channels (num_b) is less than the predefined $m a x \_b$ value, then the $\mathrm{CH}$ silently waits for the time period $\left(\max \_b-n u m \_b\right) \times 2 T_{s}$. This guarantees that member nodes can successfully derive a frequency-time correlation pattern with a constant cycle.
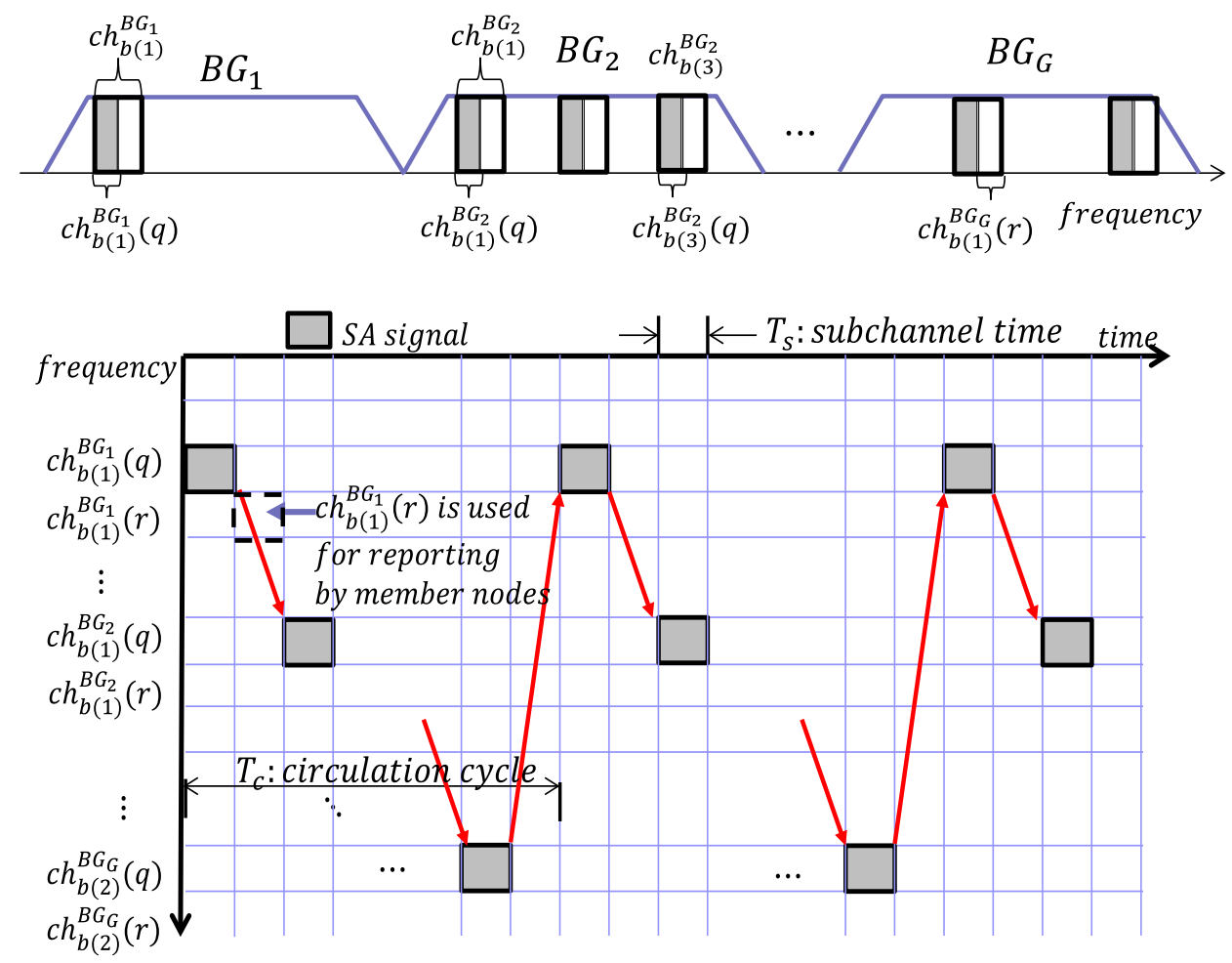

Fig. 2. System activation signal transmission. 


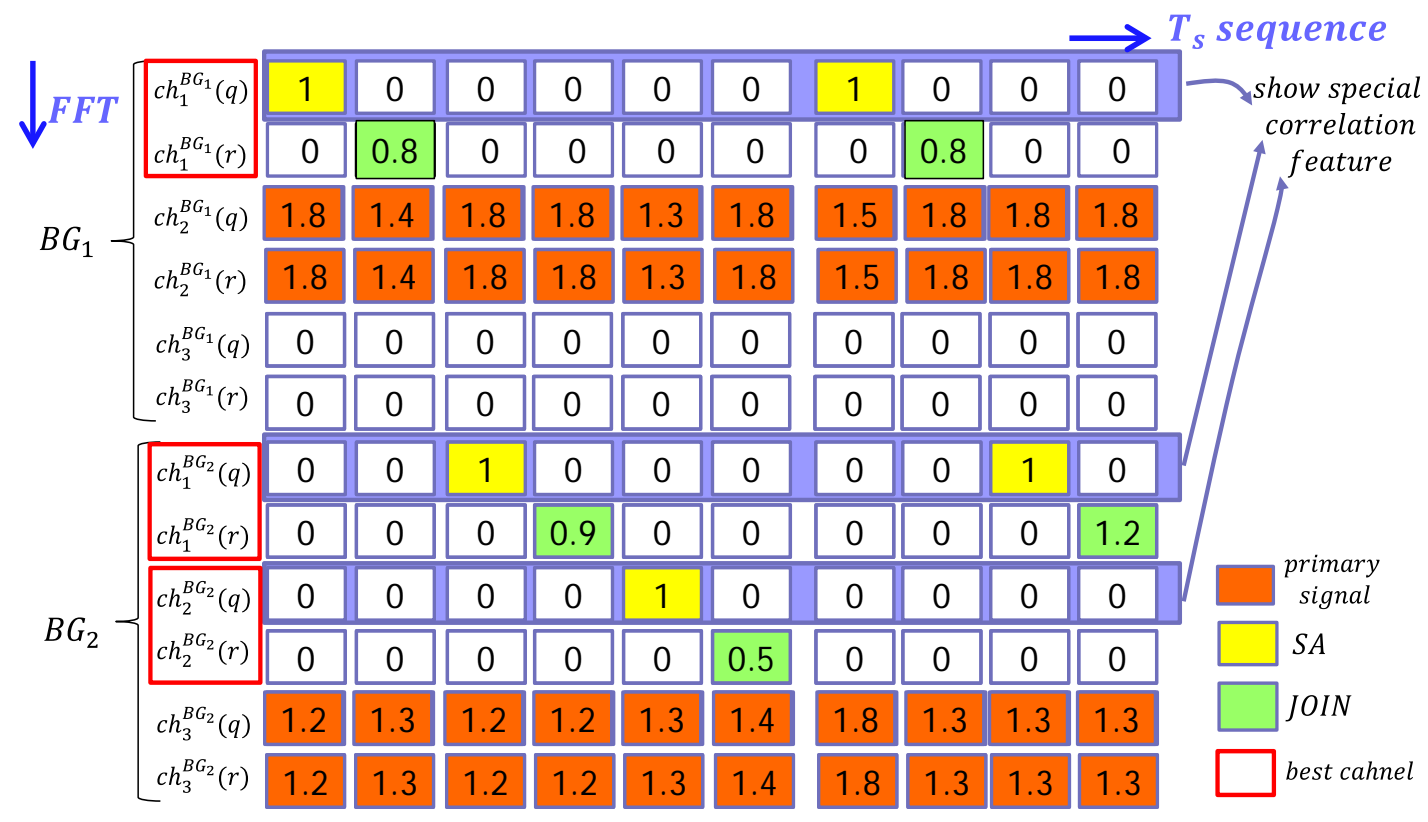

Fig. 3. Example of FFT sequences.

The proposed SA transmission scheme is designed so the FFT samples of the SA signal sequence have a special time correlation feature, so every member node can detect the broadcast channel easily and efficiently. As we can see in Fig. 2, for each request sub-channel on which the SA signal is transmitted, the FFT value time sequence of the sub-channel has a periodic pattern (period $=2 T_{S} \times \max \_b$ ). The waveform of the SA signal during $T_{S}$ is designed to correspond with a bin value of $(a+j b)$ FFF value, in which the FFT bin size is the sub-channel bandwidth and $(a, b)$ values are predetermined. Therefore, the FFT time sequence for each SA transmitted request sub-channel shows the following pattern:

$$
\operatorname{Tseq} q_{c h(q)}^{F F T}=\underbrace{\{(a+j b), 0, \cdots, 0\}}_{\text {max_cycle }}, \underbrace{\{(a+j b), 0, \cdots, 0\}}_{2 \times \text { max_b }_{-} b}, \cdots, \underbrace{\{(a+j b), 0, \cdots, 0\}}_{2 \times \text { max }_{\_} b}
$$

Fig. 3 illustrates an example scenario. In Fig. 3, the number of band groups is 2; the number of maximum best channels, max_ $b$, is 3 ; the number of channels for each band group is 3 . From among six channels, four channels are available to the $\mathrm{CH}$. Since $\max _{-} b$ is 3 , one channel from band group 1 and two channels from band group 2 are selected as best channels. The $\mathrm{CH}$ broadcasts the SA signal on each request sub-channel of the selected best channels and waits for possible join messages from member nodes on the corresponding response sub-channels. In Fig. 3, an SA signal FFT bin value of $a+j b=1.0+j 0$ is used. The sub-channels of primary detected channels show different FFF bin time sequences from that of the SA request sub-channel. 


\subsection{SA Sub-channel Detection by Member Nodes}

For fast and reliable system setup for secondary cognitive radio networks, cluster member nodes first need to find the system activation channels. Each member node periodically performs local wideband sensing and maintains its available channel list for each band group. Then, to detect the SA transmitted sub-channel, member nodes perform FFT operations for available channels. In this paper, to reduce FFT computation overhead, member nodes can optionally select one (or multiple) band group(s) with the largest number of available channels. Instead of performing FFT for all band groups, the member nodes may take FFT only for the selected band group(s). As shown in Fig. 4, band group $B G_{2}$ has the largest number of available channels (four), and $B G_{2}$ is therefore selected by the member node to perform the FFT operation.

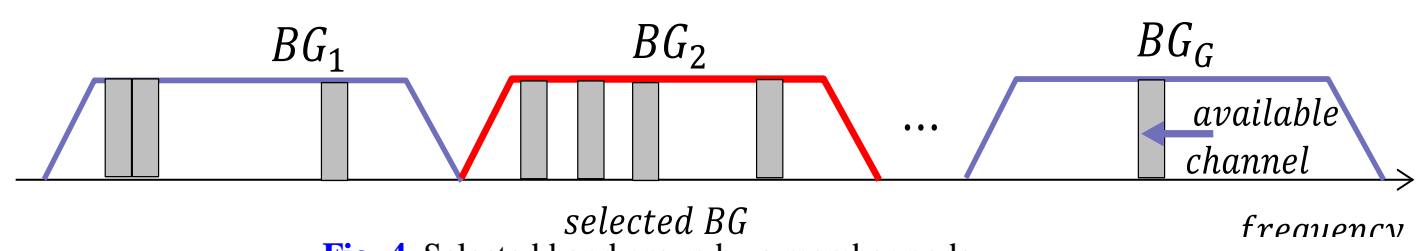

Fig. 4. Selected band group by a member node.

Since the actual received signal can be attenuated and added by additive white Gaussian noise (AWGN), and can also experience shadowing and fading effects, the received SA signal's FFT time sequence can be different from that of the transmitted sequence, as seen in (2). The FFT signal values also differ between member nodes due to the different node positions:

$$
\operatorname{Rseq}_{c h(q)}^{F F T}=\underbrace{\left\{(a+j b)^{\prime}, n, \cdots, n\right\}}_{\text {max_cycle }}, \underbrace{\left\{(a+j b)^{\prime}, n, \cdots, n\right\}}_{2 \times \max _{-} b}, \cdots, \underbrace{\left\{(a+j b)^{\prime}, n, \cdots, n\right\}}_{2 \times \max _{-} b}
$$

where, $(a+j b)^{\prime}$ is the FFT value for the received SA signal, and $n$ is the FFT value for random noise. Note that $(a+j b)^{\prime}$ in (2) for the same member node can be different due to AWGN, fading and shadowing in each measurement time.

In this paper, we do not use the received SA signal's FFT value itself as a pattern. Instead, the correlation of the FFT values is the specialized feature to detect the SA transmission sub-channel. In our proposed method, the FFT calculation interval is predetermined and is equal to sub-channel time $T_{s}$. As we can see in Fig. $\mathbf{5}$, there is no need for time synchronization for cluster initialization between cluster head and member nodes. The FFT calculation can be done any time within the $\mathrm{CH}$ sub-channel time.

Each member node calculates autocorrelation of the received signal's FFT time sequence for each of its available channels. As shown in Section 2.1, the entire band group and channel formation are known to member nodes in advance, and the SA signal is only transmitted on the request sub-channels. Therefore, member nodes only need to perform FFT for odd sub-channels (i.e., request sub-channels) of their selected band group. Also for the sub-channels of primary detected channels, member nodes do not perform correlation computations. 


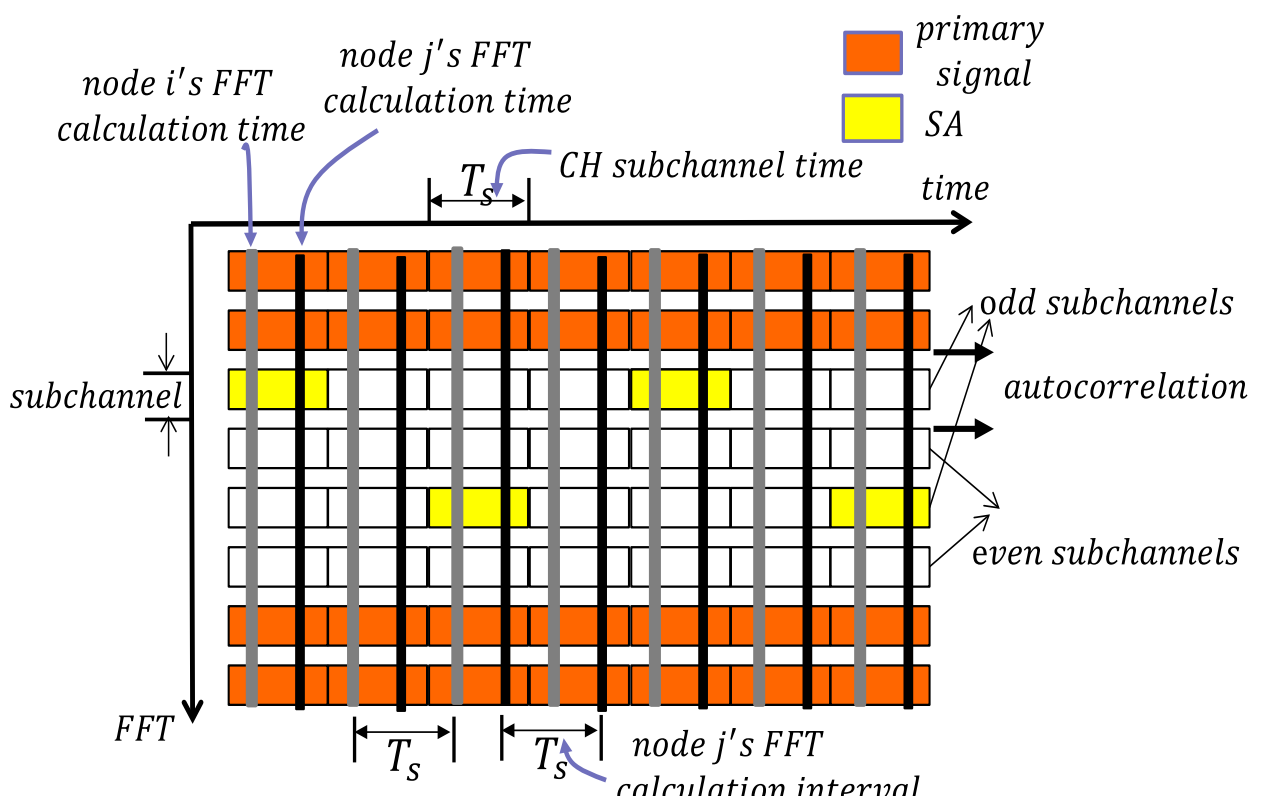

Fig. 5. Member nodes' FFT and autocorrelation calculations.

As a case study, Fig. 6 and Fig. 7 show FFT time sequences and autocorrelations for $T \operatorname{seq}_{c h(q)}^{F F T}$ of (1) ('Ideal') and $R \operatorname{se} q_{c h(q)}^{F F T}$ of (2) ('Shadowing+AWGN'). Here, $a+j b=1+\mathrm{j} 1$ is used; max_b is 3; max_cycle is 3 . As shown in Fig. 6, the FFT value sequences of the transmitted and the actual received signals are not much different due to the shadowing effect ( $\sigma=2 \mathrm{~dB}$ ) and AWGN (SNR=-5dB). In this study, SNR is the signal-to-noise ratio for the time domain signal before the FFT operation at the receiver. As shown in Fig. 7, the reference FFT sequence has a specific correlation feature, depending on $\max _{-} b$ and max_cycle.

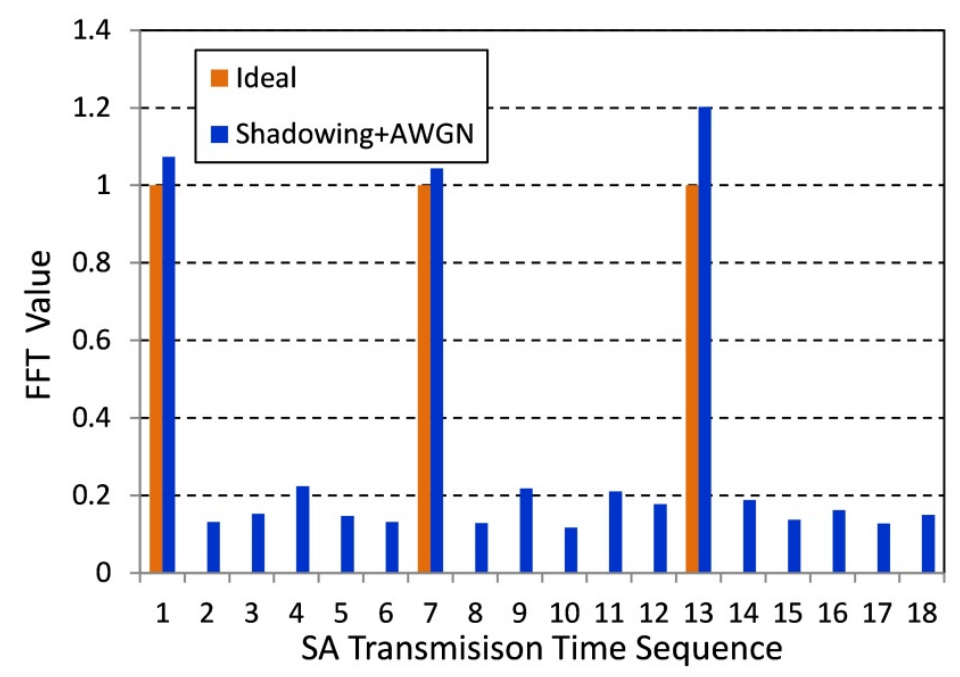

Fig. 6. FFT SA signal values. 


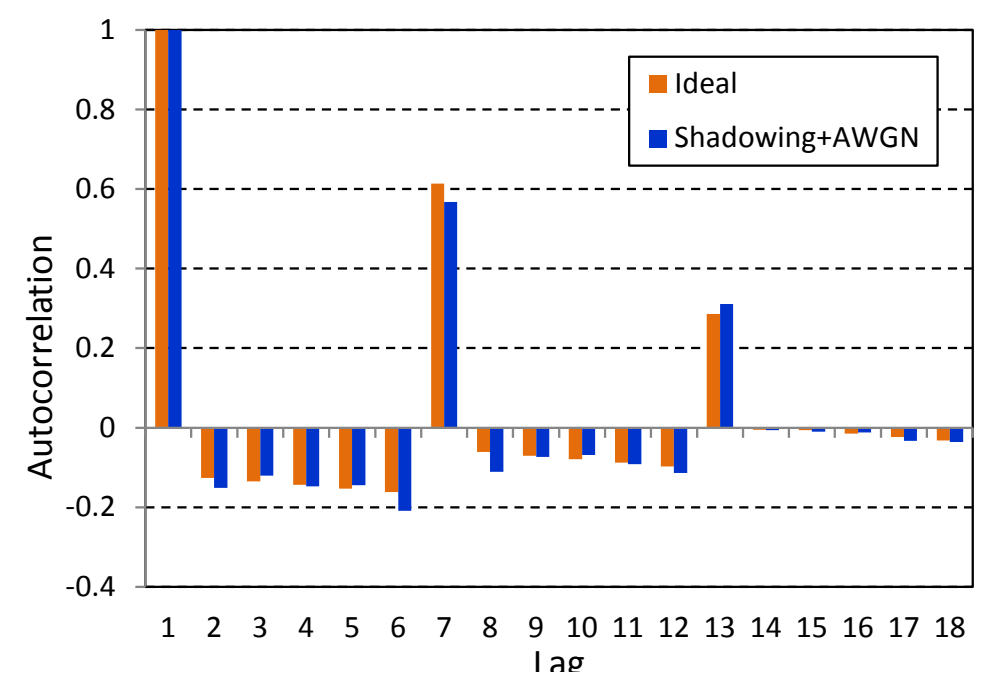

Fig. 7. Autocorrelation of the FFT sequence of the SA transmitted channel.

To determine whether on channel $m$ the $\mathrm{CH}$ sent the SA signal or not, member nodes compare the autocorrelation feature of the measured FFT bin sequence of the request sub-channel of channel $m$ with that of the reference FFT sequence. In this paper, we use two similarity decision methods: i) product-based cross-correlation matching and ii) mean squared error (MSE)-based matching. The similarity values of channel $\mathrm{m}$ from product-based cross-correlation matching and MSE-based matching are given in (3) and (4), respectively.

$$
\begin{aligned}
& \operatorname{Sim}_{-} P^{m}=\boldsymbol{R}_{\boldsymbol{m s r}}^{\boldsymbol{m}} * \boldsymbol{R}_{\text {ref }}=\frac{1}{L} \sum_{l=1}^{L} R_{m s r}^{m}[l] R_{\text {ref }}[l] \\
& S i m \_M S E^{m}=E\left[\left(R_{m s r}^{m}[l]-R_{r e f}[l]\right)^{2}\right]=\frac{1}{L} \sum_{l=1}^{L}\left(R_{m s r}^{m}[l]-R_{r e f}[l]\right)^{2}
\end{aligned}
$$

where

$$
\begin{aligned}
& R_{m s r}^{m}[l]=\text { autocorrelation of the measured FFT bin sequence for the request } \\
& \text { sub-channel of channel } m \text { with time lag } l \\
& R_{r e f}[l]=\text { autocorrelation of the predetermined reference FFT sequence pattern with } \\
& \text { time lag } l \\
& L=2 \times \text { max_b } \times \text { max_cycle }=\text { the number of autocorrelation samples }
\end{aligned}
$$

The similarity values are compared with thresholds. If $\operatorname{Sim}_{-} P^{i} \geq T h_{p}$ (for product-based cross-correlation matching) or $\operatorname{Sim}_{-} M S E^{i} \geq T h_{m s e}$ (for MSE-based matching), then member nodes consider the SA signal to be sent on channel $i$. If there is no matched channel, then member nodes select a new BG and perform the initialization process again.

\section{Cluster Join and Common Channel Decision Notification Procedure}

\subsection{Member Node Join Procedure}

At a member node, if autocorrelation of a certain FFT bin time sequence on a request 
sub-channel matches the predefined correlation pattern, then the member node sends a JOIN request to the $\mathrm{CH}$ using the corresponding response sub-channel. In other words, when the FFT correlation matched by a sub-channel is $c h_{m}^{B G_{g}}(q)$, the JOIN request is sent on $c h_{m}^{B G_{g}}(r)$ where $g$ is the selected band group by the member node and $m$ is the SA detected channel.

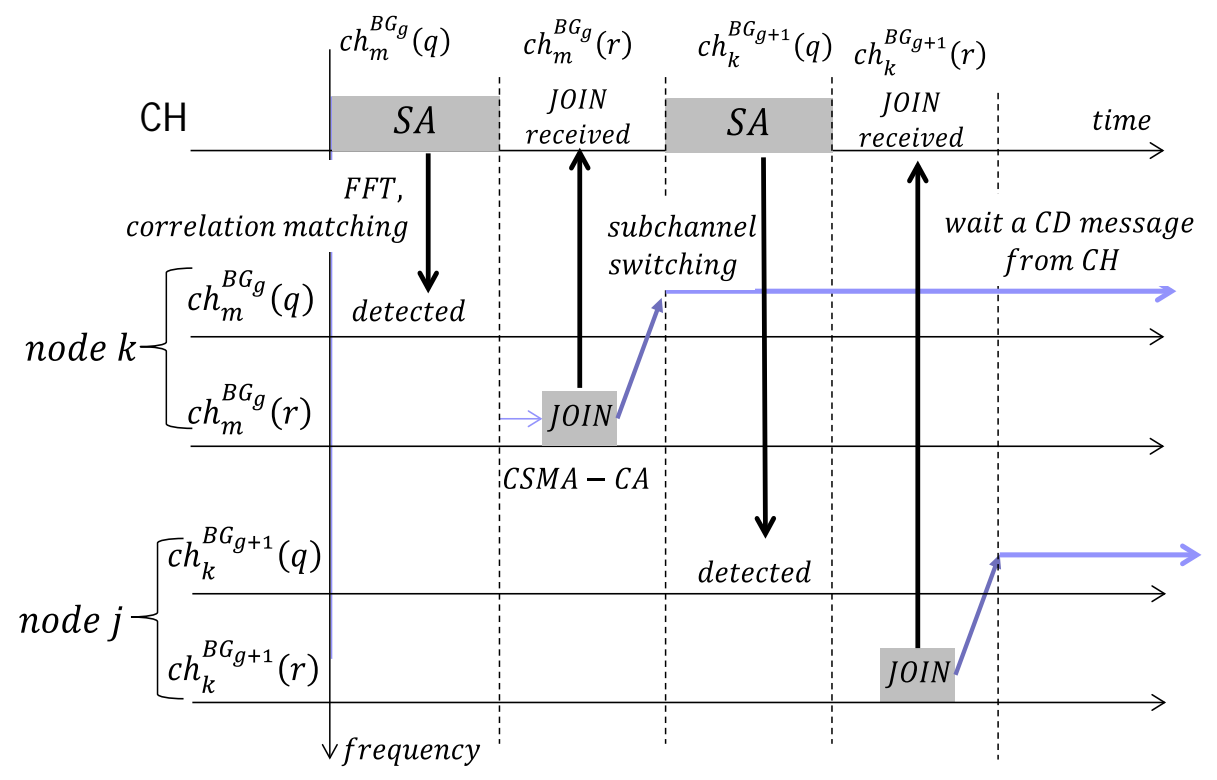

Fig. 8. Member node JOIN message transmission.

Fig. 8 shows the member node's JOIN message transmission procedure. As shown in Fig. 8, the SA signal sent by the $\mathrm{CH}$ on the $c h_{m}^{B G_{g}}(q)$ sub-channel is detected by node $k$, and a JOIN request is sent on the $c h_{m}^{B G_{g}}(r)$ sub-channel in response. After the $\mathrm{CH}$ sends the SA signal on the request sub-channel, it switches to the response sub-channel and waits for possible JOIN messages from the member nodes. To avoid possible JOIN request collisions between member nodes, carrier sense multiple access with collision avoidance (CSMA/CA) can be used. In the JOIN request, all available channel lists of the member node and the channel state information are included, not only for the selected band group $g$, but also all other band groups. After sending a JOIN request, the member node immediately switches to the $\operatorname{ch}_{m}^{B G_{g}}(q)$ sub-channel and simply listens to the channel and waits for the channel decision (CD) message without further FFT operation.

\subsection{Common Channel Decision Procedure}

After broadcasting the SA signal on the $c h_{m}^{B G_{g}}(q)$ sub-channel, the $\mathrm{CH}$ switches to the $c h_{m}^{B G_{g}}(r)$ sub-channel and listens during sub-channel time $T_{s}$ to receive JOIN messages from the cluster member nodes. Then, the $\mathrm{CH}$ switches to the next request sub-channel and transmits the SA signal. If the $\mathrm{CH}$ has received enough JOIN messages from member nodes, then it terminates transmission of the SA signal. The $\mathrm{CH}$ determines the common channel set to use for data transmissions between cluster member nodes. The optimal common channel set is computed using the channel information in the received JOIN messages. 
The CH broadcasts a CD message on each request sub-channel $c h_{m}^{B G_{g}}(q), \forall g, m \in \boldsymbol{M}_{J}^{g}$ sequentially. $\boldsymbol{M}_{J}^{g}$ is a set of channels from JOIN messages received from band group $g$. The CD message includes a common data channel set to use, a hopping pattern (if frequency hopping is used), and an operation time schedule, and lists all member node IDs that successfully sent JOIN requests.

Fig. 9 shows an example scenario of a JOIN request. Suppose that member node $k$ sent a JOIN request and waits for a CD message from $\operatorname{ch}_{m}^{B G_{g}}(q)$. If the node has successfully received the CD message, but the node ID of node $k$ is not included in the CD message, then node $k$ sends a JOIN request again on the following $c h_{m}^{B G_{g}}(r)$ sub-channel. At the next $c h_{m}^{B G_{g}}(q)$ sub-channel time, the $\mathrm{CH}$ announces the successful membership join results to member node $k$. When node $k$ is not able to receive the CD message during the predetermined time, $T_{C D}$, then it sends a JOIN request on the other SA detected channel.

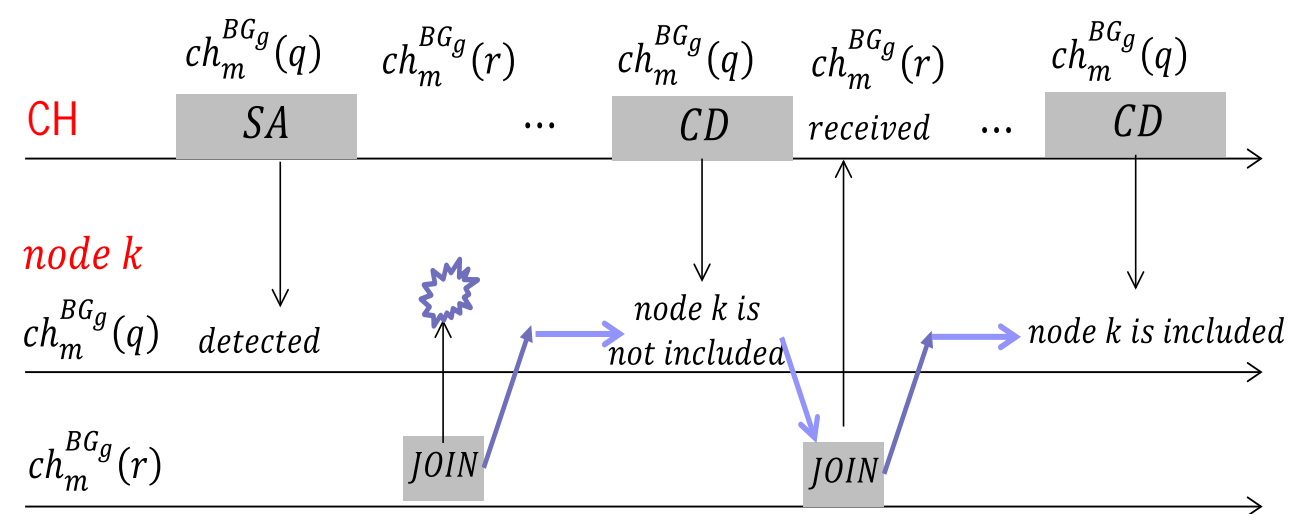

Fig. 9. Channel decision notification and JOIN retransmission.

Algorithm1 and Algorithm2 show the pseudo codes for cluster head and member node behaviors, respectively.

Algorithm1: Pseudo code of cluster head behavior.

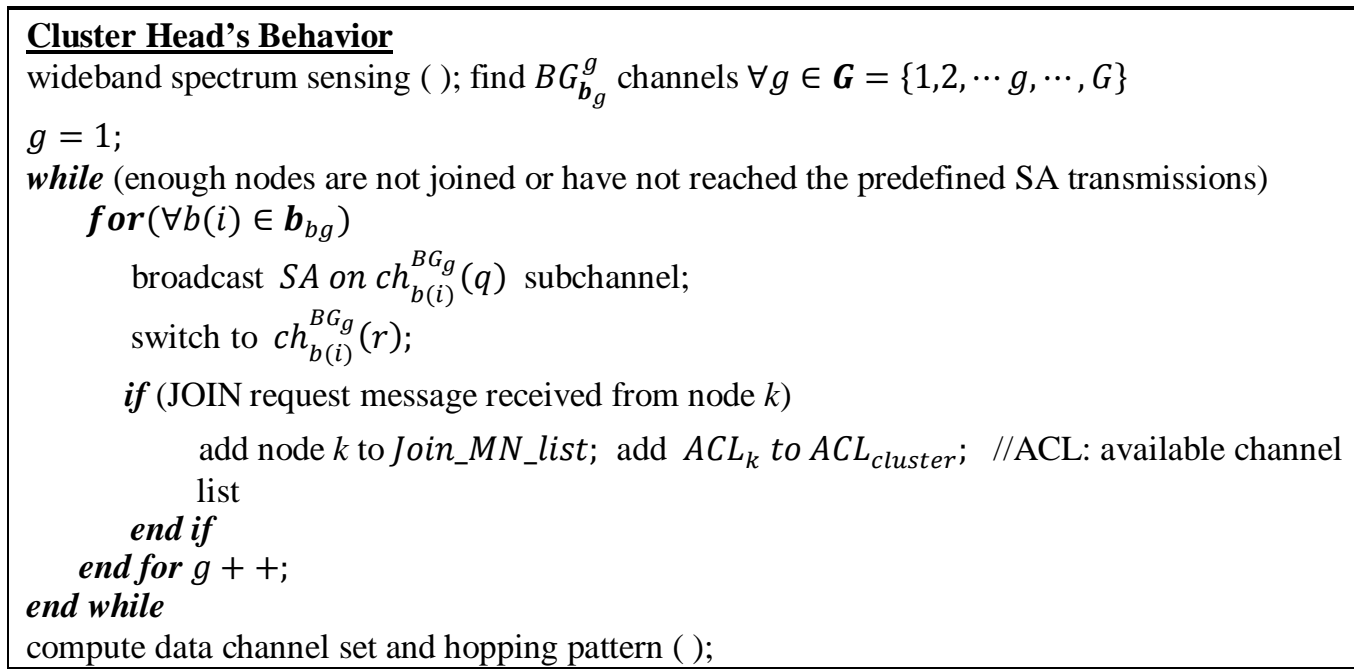




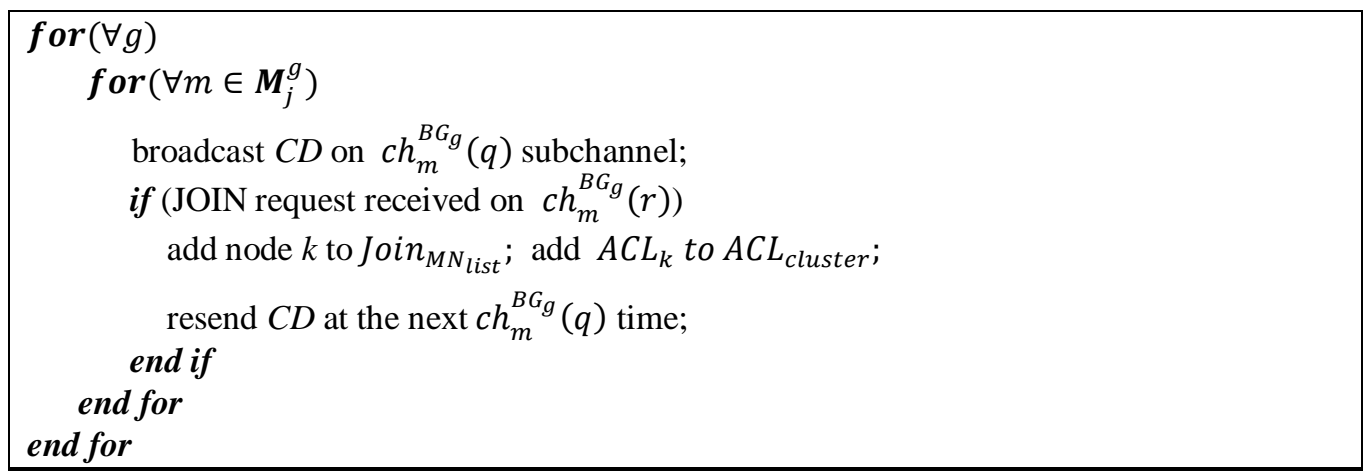

Algorithm2: Pseudo code of member node behavior.

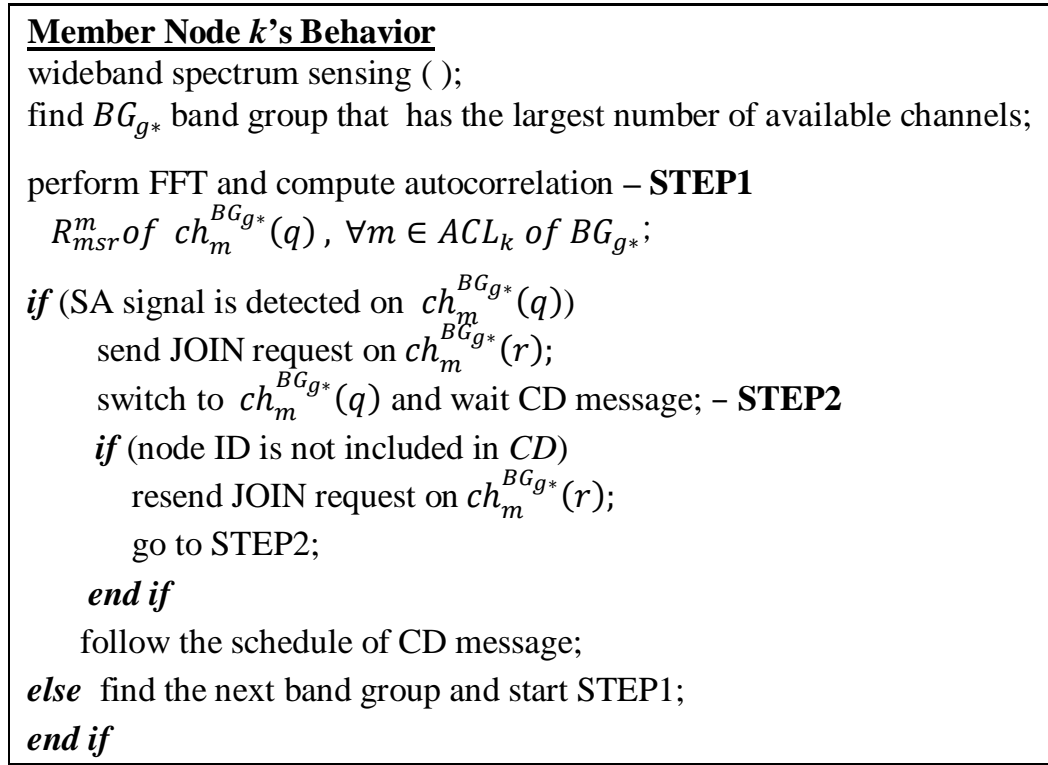

\section{Simulation Results}

In this section, we evaluate the performance of the proposed common channel initialization protocol. The maximum rendezvous time, correlation matching values, false-recognition and misrecognition ratios and JOIN message delivery delay are compared for various scenarios. In Table 1, the parameters used for the simulations are given.

Table 1. Parameters used for simulations

\begin{tabular}{ll}
\hline Parameter & Value \\
\hline Number of SA transmission channels $\left(N_{C H}\right)$ & $5 \sim 30$ \\
Number of member nodes & $10 \sim 40$ \\
SNR & $-10 \mathrm{~dB} \sim+10 \mathrm{~dB}$ \\
Speed of member nodes & $3 \mathrm{~km} / \mathrm{h}$ \\
Shadowing standard deviation $\sigma$ & $0 \mathrm{~dB} \sim 4 \mathrm{~dB}$ \\
max_cycle & $3,4,5$ \\
max_b & 3 \\
\hline
\end{tabular}


As we introduced in Section 1, there are sequence-based and group-based rendezvous protocols for dynamic spectrum access. For fast secondary system initialization, the required maximum rendezvous time should be small. In this section, the proposed mechanism is evaluated and compared with the sequence-based method [8] and group-based method [12] in terms of the required rendezvous time for secondary system initialization.

In the sequence-based rendezvous protocol of [8], $\mathrm{CH}$ and a member node need to randomly change their hopping channels to achieve hopping channel synchronization. In addition, to organize an ad-hoc CR network, $\mathrm{CH}$ should perform the initialization procedure with each member node separately. According to the analysis of [8], the total required rendezvous time for the sequence-based method is given as in (5).

$$
T_{\text {total }}^{\text {seq }}=T_{S} \times N_{M} \times \frac{N_{C H}^{4}+2 N_{C H}^{2}+6 N_{C H}-3}{3 N_{C H} \times\left(6 N_{C H}+1\right)}
$$

where, $T_{S}$ is the hopping time (corresponding to the sub-channel time in the proposed method) $N_{M}$ is the number of member nodes; $N_{C H}$ is the number of hopping channels (corresponding to the number of SA channels in the proposed method).

In the group-based cyclic-listening initial setup method of [12], each member node selects one channel from its available channels and waits for a $\mathrm{CH}$ system activation message for a time period of at least $T_{S} \times N_{C H}$. $T_{S}$ is the system activation (SA) message transmission time, and $N_{C H}$ is the number of $\mathrm{CH}$ activation message transmission channels. If a member node does not receive an SA message, then it switches to the next channel and waits again. This is done for $N_{C H}$ channels, so that the total required rendezvous time of the group-based method of [12] is computed with (6).

$$
T_{\text {total }}^{\text {group }}=T_{S} \times N_{C H}^{2}
$$

In the proposed method, using an FFT operation, each member node can listen to all available channels at the same time. When the required number of cycles for a pattern is max_cycle, the total required SA transmission time of the proposed method is given with (7).

$$
T_{\text {total }}^{\text {proposed }}=T_{S} \times \text { max_cycle } \times N_{C H}
$$

Fig. 10 shows a comparison of the total required rendezvous time. For the proposed method, max_cycle is set to 3,4 and 5. As we can see, the proposed method is not sensitive to the number of SA transmission channels and the number of member nodes. The required time for system initilization of the proposed method is much less than those of sequence and group -based methods. 


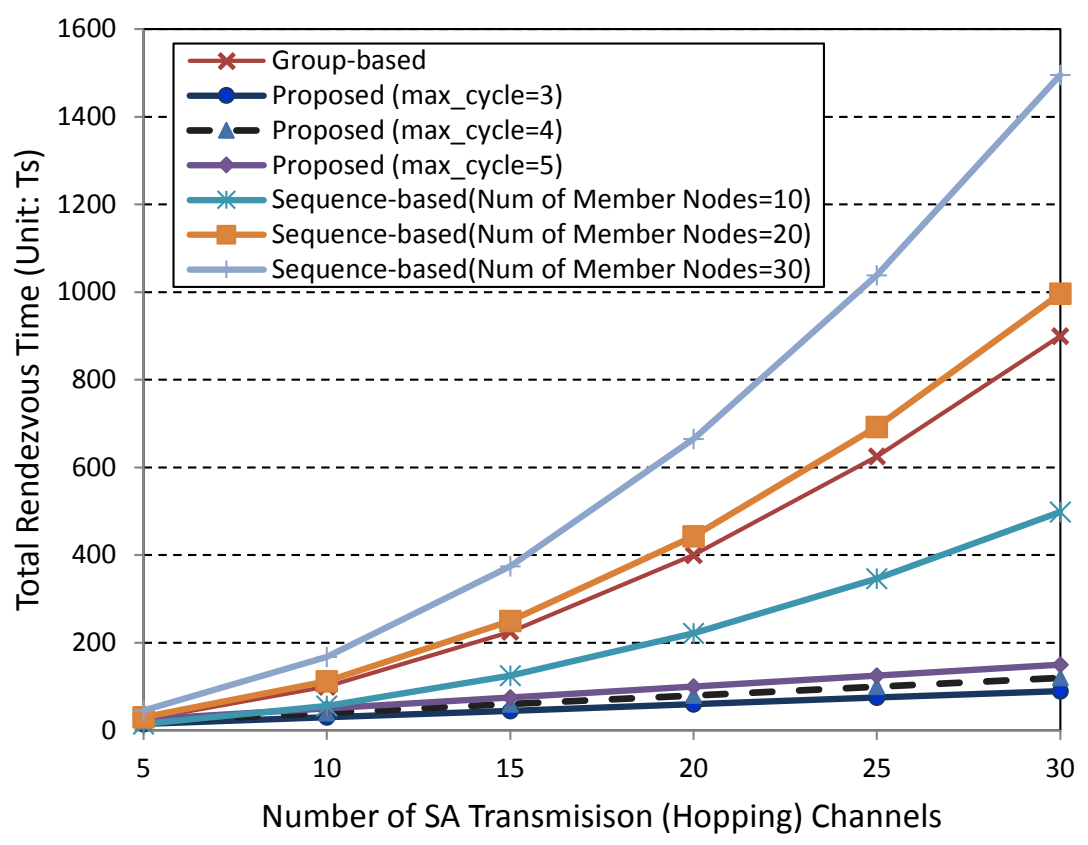

Fig. 10. Required rendezvous time for system initialization.

When member nodes transmit JOIN message to $\mathrm{CH}$ at the same time on the same sub-channel, they may contend to take a response sub-channel. If the number of contending member nodes is increasing, then JOIN message collision ratio and the message transmission delay can be also increasing. In Fig. 11, we evaluated the JOIN message transmission delay. For the simulation study $T_{S}$ is set to $\max \left[T_{S A},\left(T_{\text {backoff }}+T_{\text {JOIN }}\right)\right] . T_{S A}$ is the SA transmission time; $T_{\text {JOIN }}$ is the JOIN message transmission time; $T_{\text {backoff }}$ is the maximum backoff time in a sub-channel time channel access. In this example case, during $T_{s}$, only one message can be sent. For the different network conditions, we measured the required number of response $T_{S}$ slots for all member node's successful JOIN transmissions. The number of backoff mini-slots is eight. The average number common available channels between $\mathrm{CH}$ and member nodes varies from 5 to 30 , in which we assume that each member node tries to send JOIN message using one of SA transmission channels. The number of member nodes varies from 10 to 40. As we can see in Fig. 11, as the number of member nodes increasing for the given number of common available channels between $\mathrm{CH}$ and member nodes, the required number of response sub-channel slots for successful transmissions of JOIN message is also increasing due to deferring the JOIN transmission and possible message collisions. 


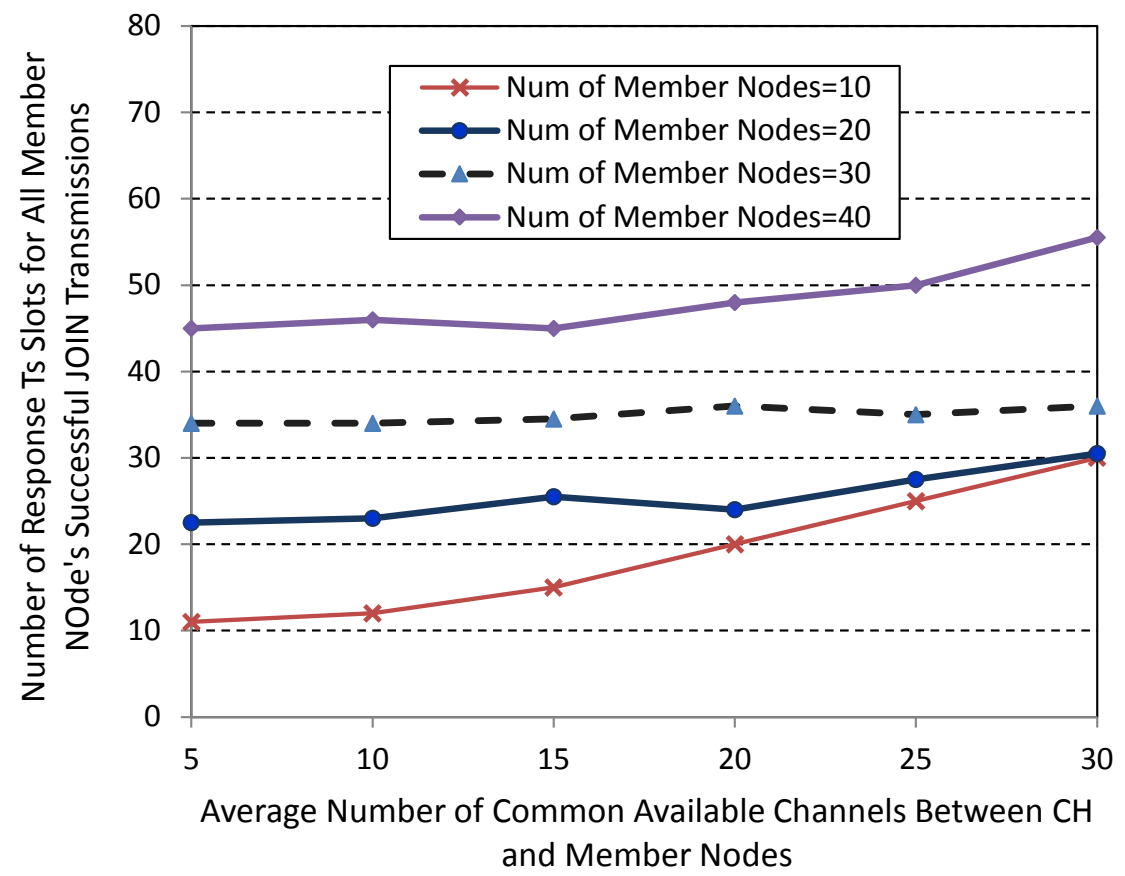

Fig. 11. The required number of response sub-channel slots for successful transmissions of JOIN message.

To evaluate the effectiveness of the proposed correlation-based SA transmission channel detection under various wireless channel conditions, we adopted the Friss path loss model and shadowing effect, as expressed in (8):

$$
\left[\frac{P_{r}(d)}{P_{r}\left(d_{0}\right)}\right]_{d B}=-10 \beta \log \left(\frac{d}{d_{0}}\right)+X_{\sigma}
$$

where $d$ is the distance between the member node and the cluster head; $P_{r}(d)$ denotes the received signal power at distance $d ; \beta$ is the path loss exponent; $P_{r}\left(d_{0}\right)$ is the received power at the close-in reference distance $d_{0} ; X_{\sigma}$ is a random variable with Gaussian distribution with a zero mean and a standard deviation of $\sigma \mathrm{dB}$. In the following simulation, member nodes move away from the cluster head at a speed of $3 \mathrm{~km} / \mathrm{h}$. In this simulation study, max_b is three channels and max_cycle is set to 3 . The SA circulation cycle $\left(T_{c}\right)$, which represents the periodic SA transmission interval on a specific sub-channel, is six sub-channel times because $T_{c}=\left(T_{s} \times 2\right) \times \max \_b$.

Fig.12 shows the product-based average cross-correlation matching values between the predefined autocorrelation pattern and the measured autocorrelation under various wireless channel conditions when an SA signal is transmitted. We can see that as the SNR conditions worsen and larger shadowing variations result in lower average cross-correlation matching values. However, it is also shown that even in the worst case, i.e., $-10 \mathrm{~dB}$ SNR and a $4 \mathrm{~dB}$ shadowing standard variation, the computed average cross-correlation value is higher than 0.72 . At $-10 \mathrm{~dB} \sim 10 \mathrm{~dB}$ SNR and under $0 \mathrm{~dB}, 1 \mathrm{~dB}$, and $2 \mathrm{~dB}$ shadowing standard variation conditions, the computed average cross-correlation matching values are always higher than 0.83 , which is large enough to detect the SA transmission channel at the member nodes. As we 
mentioned in Section II, SNR is the signal-to-noise ratio for the time domain signal before the FFT operation at the receiver. In our simulation study, we confirmed that the typical values of cross-correlation between the predefined signal pattern and some other signal types, such as constant primary signals or random signals under various wireless channel conditions, were about 0.55 .

Fig. 13 presents the average MSE correlation matching performance of the proposed scheme as a function of SNR. It represents the average squared difference between the pre-defined autocorrelation pattern and the measured autocorrelation under different SNR conditions. As we can see, worse SNR conditions and higher shadowing variations result in higher average MSE matching values, whereas higher SNR conditions and lower shadowing variations result in smaller average MSE matching values. We also confirmed that the typical values of MSE correlation matching for some other signal types are several times larger than those of Fig. 13.

We compared the average correlation matching values for different types of signals. Signal type 1 represents a constant primary signal with AWGN, in which a constant signal waveform from a primary system is transmitted on the member node's available channel. Signal type 2 is a primary signal with random waveform. Signal type 3 is AWGN-only, and signal type 4 indicates that the SA signal is transmitted on a channel with AWGN and shadowing. Fig. 14 and Fig. 15 are average correlation matching value comparisons for product-based and MSE-based, respectively. Here the SNR is fixed at $0 \mathrm{~dB}$, and the shadowing effect is fixed at $2 \mathrm{~dB}$.

For product-based matching, the average cross-correlation matching values for signal type 1 (constant primary signal with AWGN) and type 2 (random signal) and type 3 (AWGN only) are all less than 0.66. For signal type 4 (SA signal with AWGN and shadowing), the matching value with our proposed method is about 0.98. For MSE-based matching, the average matching values for signals of type 1 , type 2 and type 3 are all greater than 0.05 , whereas for signal type 4 , the matching value is about 0.003 . The lower average MSE-matching value indicates better matching with the reference SA pattern. It is evident in the figures that by using the SA signal activation procedure, the SA signal transmitted by the $\mathrm{CH}$ can easily be distinguished at the member nodes from other signal types, which will eventually reduce the risk of false recognition and misrecognition of the SA signal, and a fast and reliable secondary system initialization procedure is achieved in CR ad-hoc networks.

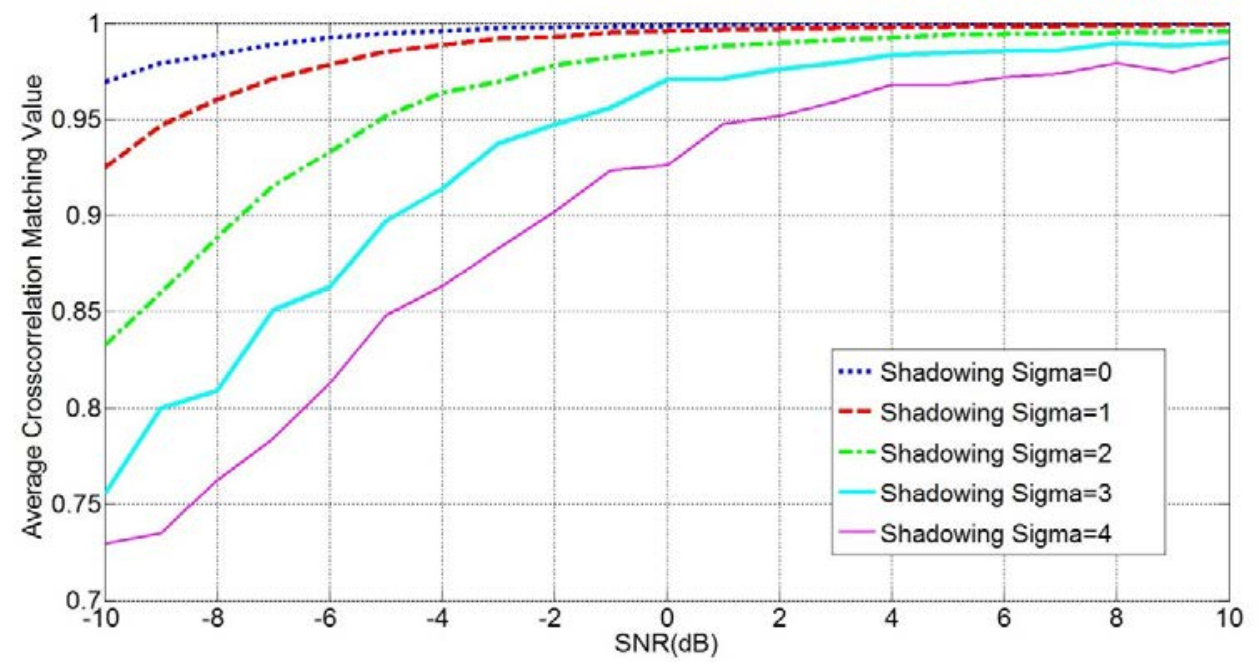

Fig. 12. Average product-based cross-correlation matching values. 


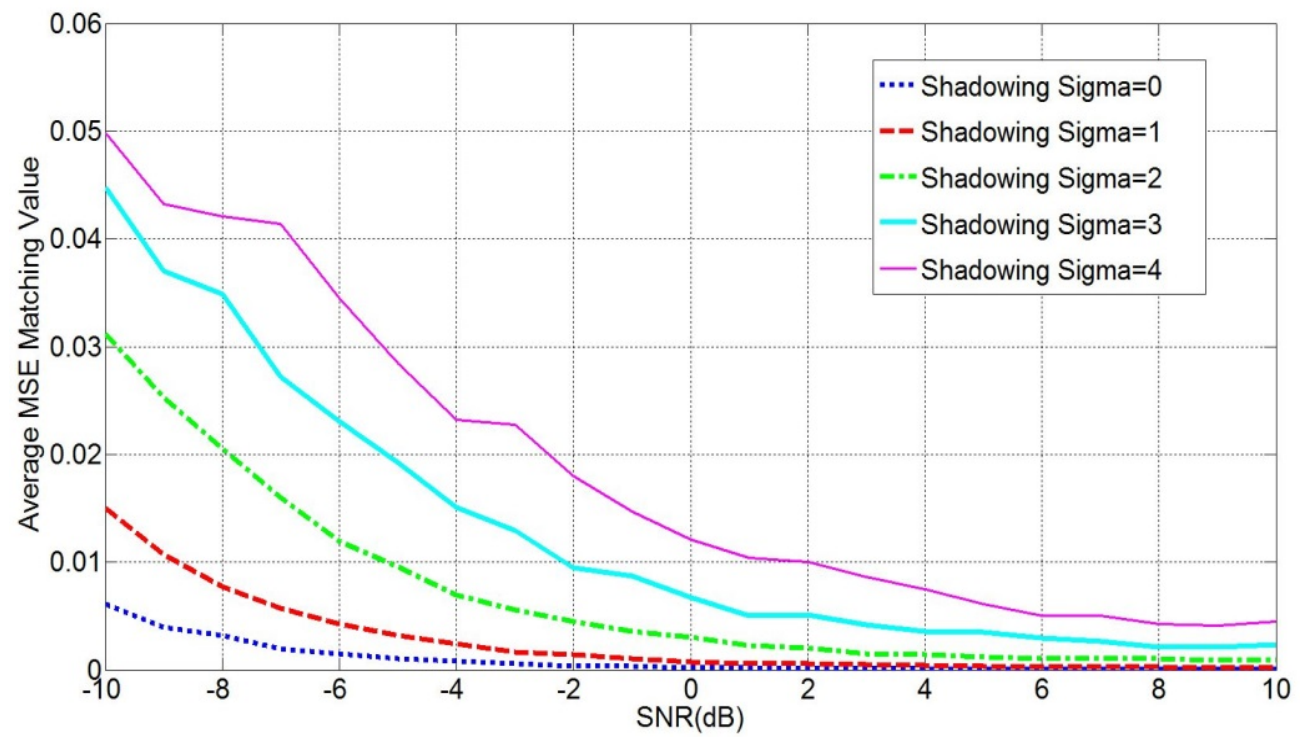

Fig. 13. Average MSE-based matching values.

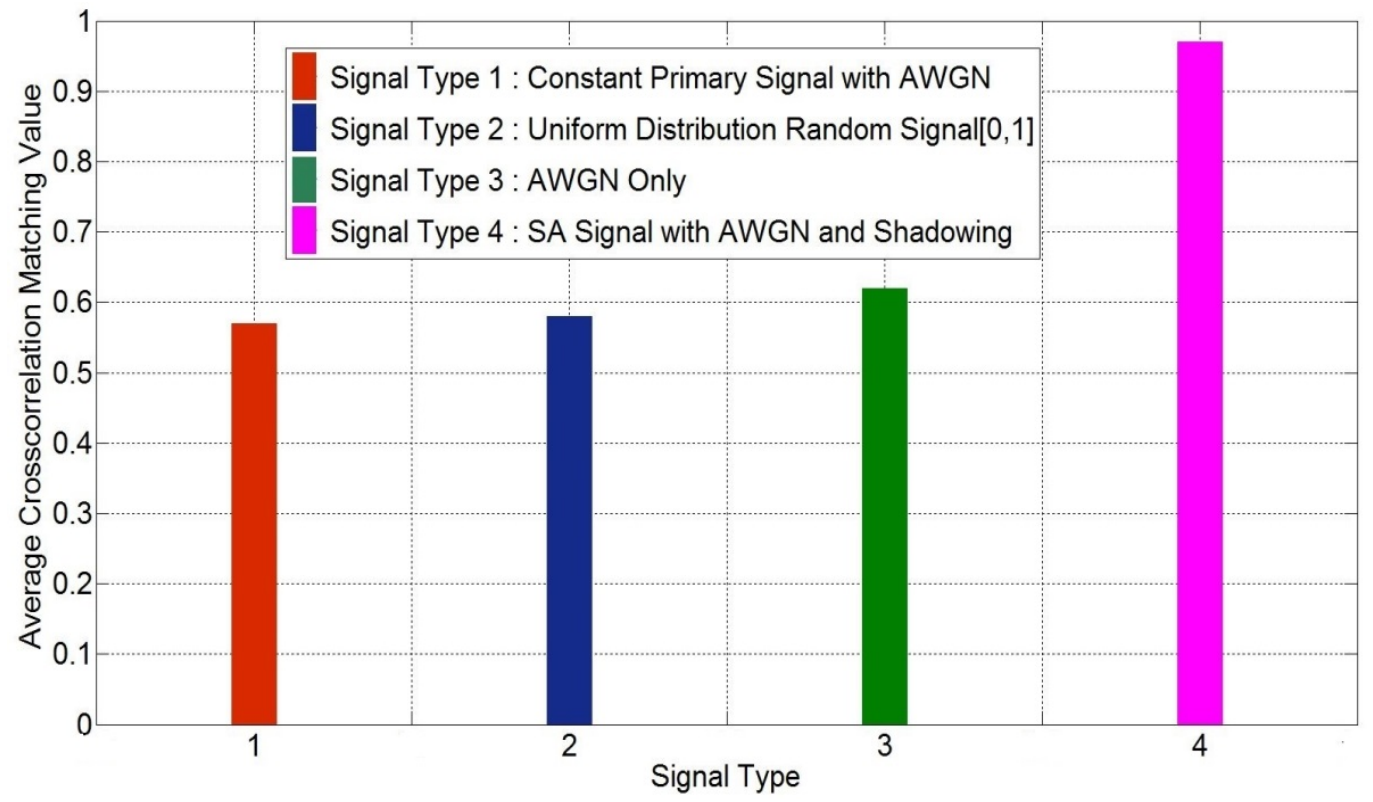

Fig. 14. Product-based average cross-correlation matching values for different signal types. 


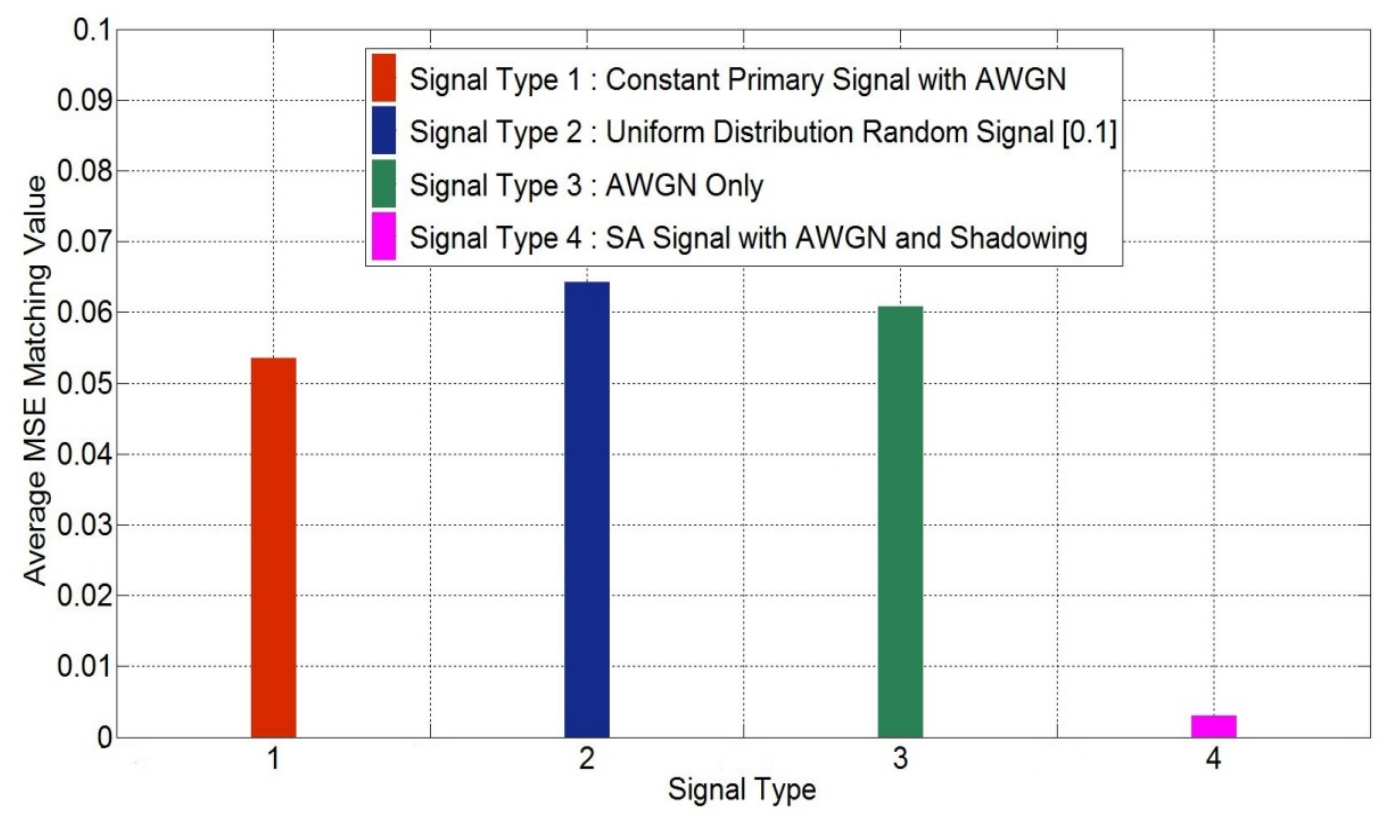

Fig. 15. Average MSE-matching values for different signal types.

For the AWGN and SA signals, Fig. 16 and Fig. 17 display the probability density function (PDF) of the matching values for product-based and MSE-based methods, respectively. Fig. 16(a) and Fig. 17(a) show the PDF of matching values calculated between the AWGN and the predefined pattern. Here the mean matching values for both methods are 0.63 and 0.06 , which coincide with the results for signal type 3 (AWGN only) of Fig. 14 and Fig. 15. In Fig. 16(b) and Fig. 17(b) SNR is set to $0 \mathrm{~dB}$, and we obtained PDF graphs for different shadowing conditions. The larger shadowing effect results in the wider matching value distribution. As shown in Fig. 16 and Fig. 17, the matching value variance for type 3 (AWGN only) and type 4 (SA signal with AWGN and shadowing effect) of the MSE-based method is a little larger than that of the product-based method, even though the MSE-based method shows a greater difference in average matching values between type 3 and type 4 signals, as in Fig. 15.

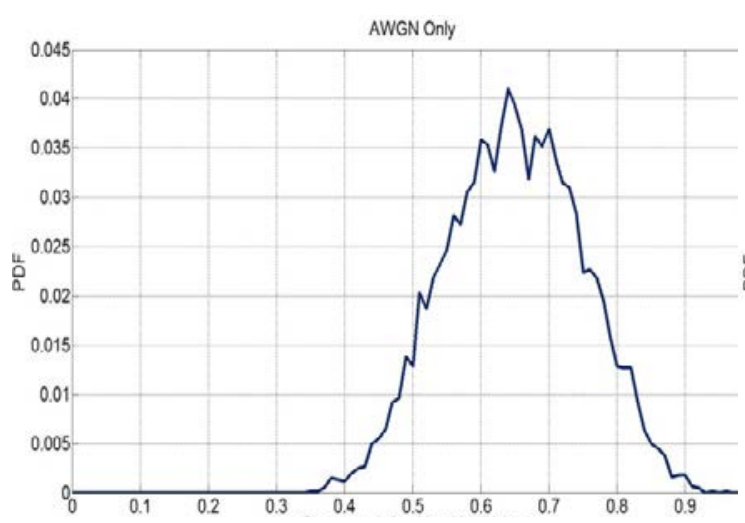

(a) AWGN only

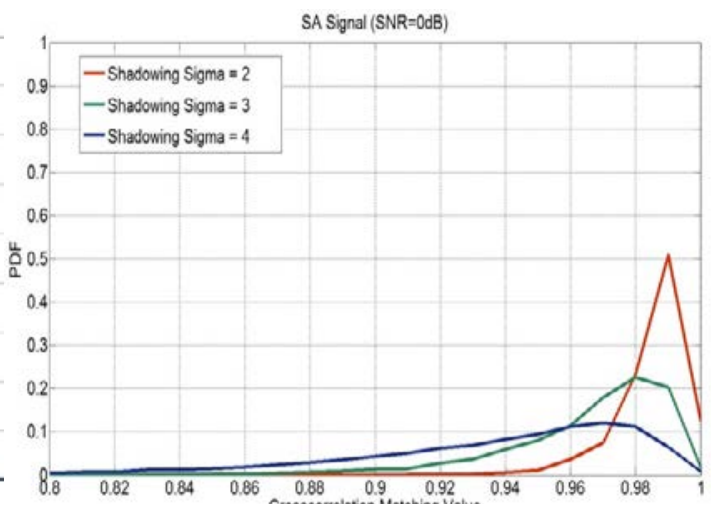

(b) SA signal with AWGN and shadowing

Fig. 16. The PDF of product-based cross-correlation matching values for different signal types. 


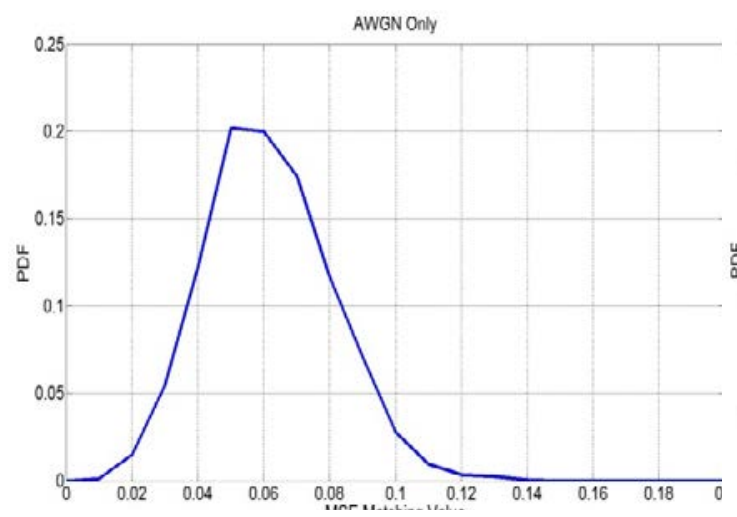

(a) AWGN only

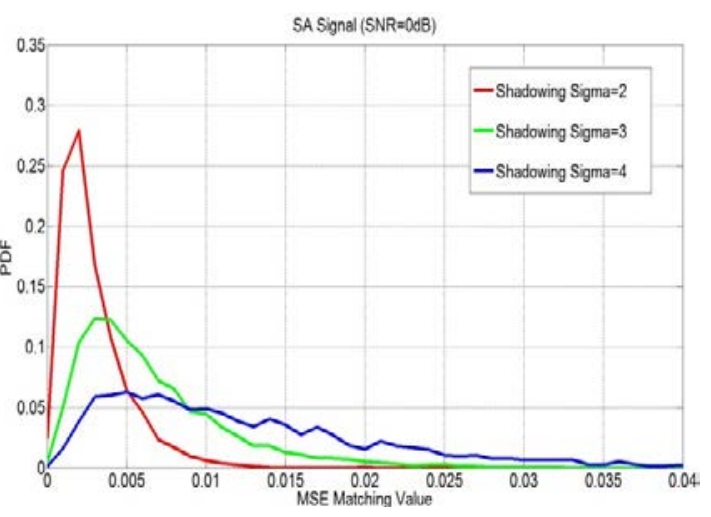

(b) SA signal with AWGN and shadowing

Fig. 17. The PDF of MSE-matching values for different signal types.

In fact, the results of Fig. 16 and Fig. 17 indicate that there are possibilities for false-recognition of noise channel to SA channel, and of misrecognition of SA channel to noise channel due to the not ignorable matching value variance. Therefore, in this simulation study, we evaluated our proposed scheme in terms of false-recognition ratio (FR) and misrecognition ratio (MR) corresponding to a given threshold for both the product-based and MSE-based cases. We also see a tradeoff between the false-recognition ratio and misrecognition ratio. In this paper, the false-recognition ratio and misrecognition ratio are expressed in (9) and (10), respectively, where the $\mathrm{CH}$ did not transmit the SA signal on a channel, but a member node determined the channel to be an SA channel.

$$
\begin{aligned}
F R & =\operatorname{Prob}\left(D_{1} \mid H_{0}\right) \\
M R & =\operatorname{Prob}\left(D_{0} \mid H_{1}\right)
\end{aligned}
$$

where

$\mathrm{D}_{1}$ : At the member node, the received signal is determined as an SA signal.

$H_{0}$ : SA signal is not transmitted on the channel by the $\mathrm{CH}$.

$D_{0}$ : At the member node, the received signal is not determined to be an SA signal.

$H_{1}$ : SA signal is transmitted on the channel by the $\mathrm{CH}$.

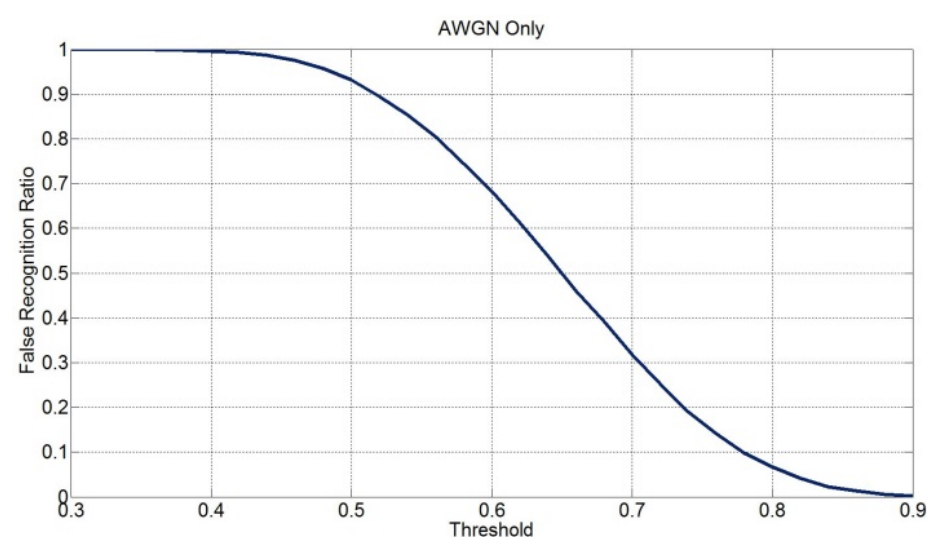

(a) False recognition ratio of noise as an SA signal 


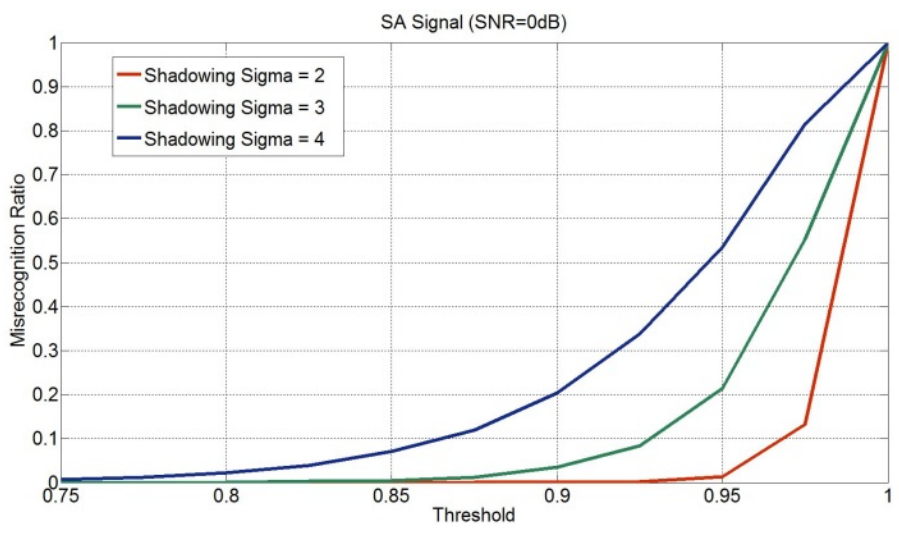

(b) Misrecognition ratio of an SA signal as another signal

Fig. 18. The product-based false-recognition ratio and misrecognition ratio for different signal types.

Fig. 18(a) and Fig. 19(a) show false recognitions of noise as an SA signal in the AWGN-only case, according to product-based and MSE-based methods, respectively. We can see that the false recognition ratio decreases as the product-based threshold increases. For a threshold value of 0.85 , the false recognition ratio is less than 5\%. In MSE-based cases, the false recognition ratio increases as the MSE-based threshold increases. For a threshold value of 0.03, the false recognition ratio is less than 5\%. Fig. 18(b) and Fig. 19(b) show misrecognition of an SA signal as a signal other than an SA signal. We fixed the SNR at OdB and changed shadowing sigma to $2 \mathrm{~dB}, 3 \mathrm{~dB}$ and $4 \mathrm{~dB}$. As we can see, for the product-based method, by increasing the threshold, the misrecognition ratio also increases, whereas when the MSE-based method increases the threshold value, the misrecognition ratio decreases. When shadowing sigma is $3 \mathrm{~dB}$, for a product-based threshold value of 0.9 , the misrecognition ratio is around $5 \%$, and for an MSE-based threshold value of 0.018 , the misrecognition ratio is around $5 \%$.

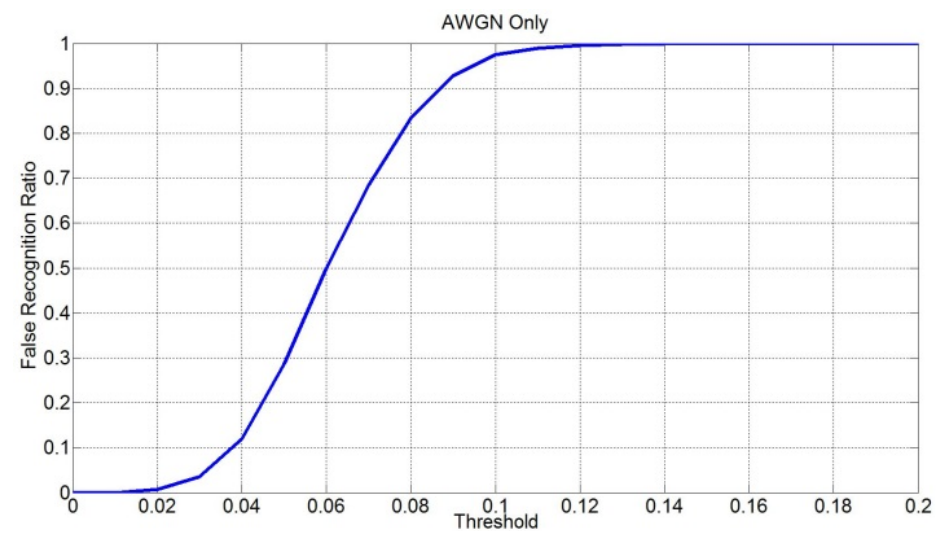

(a) False recognition ratio of noise as an SA signal 


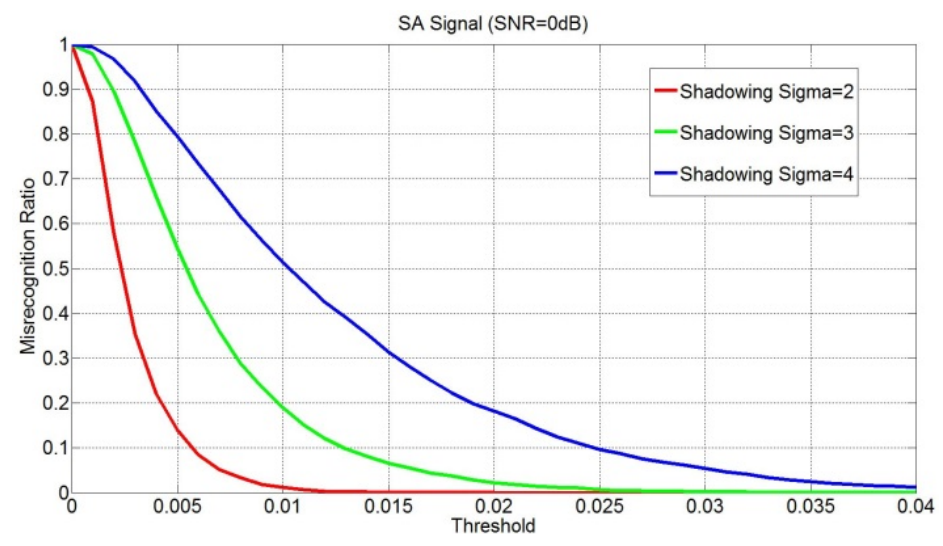

(b) Misrecognition ratio of an SA signal as another signal

Fig. 19. The MSE-based false-recognition ratio and misrecognition ratio for different signal types.

As we can see in Fig. 18 and Fig. 19, there is a tradeoff between the false-recognition ratio and misrecognition ratio. To decrease the false-recognition ratio, when we increase the product-based threshold (in MSE-based, decrease the threshold), it results in an increase in the misrecognition ratio. Therefore, in practice we need to first determine a target ratio for either false recognition or misrecognition. Then the corresponding threshold can be derived to meet the target ratio. In Fig. 20, we first determined target false recognition ratio (from 5\% to 20\%), and the corresponding threshold is derived. With the derived thresholds for both product-based and MSE-based methods, we obtained the misrecognition ratios. In this experiment, SNR is $0 \mathrm{~dB}$ and shadowing sigma varies from $2 \mathrm{~dB}$ to $4 \mathrm{~dB}$. For a $5 \%$ target false-recognition ratio, both methods show misrecognition ratios of less than $4 \%$ for different shadowing conditions. For a $10 \%$ target false-recognition ratio, they show misrecognition ratios of less than $2 \%$. For the given conditions, the misrecognition ratio of the product-based method is less than that of the MSE-based method. This is caused by the fact that the matching value variance of the MSE-based method is larger than the product-based method.

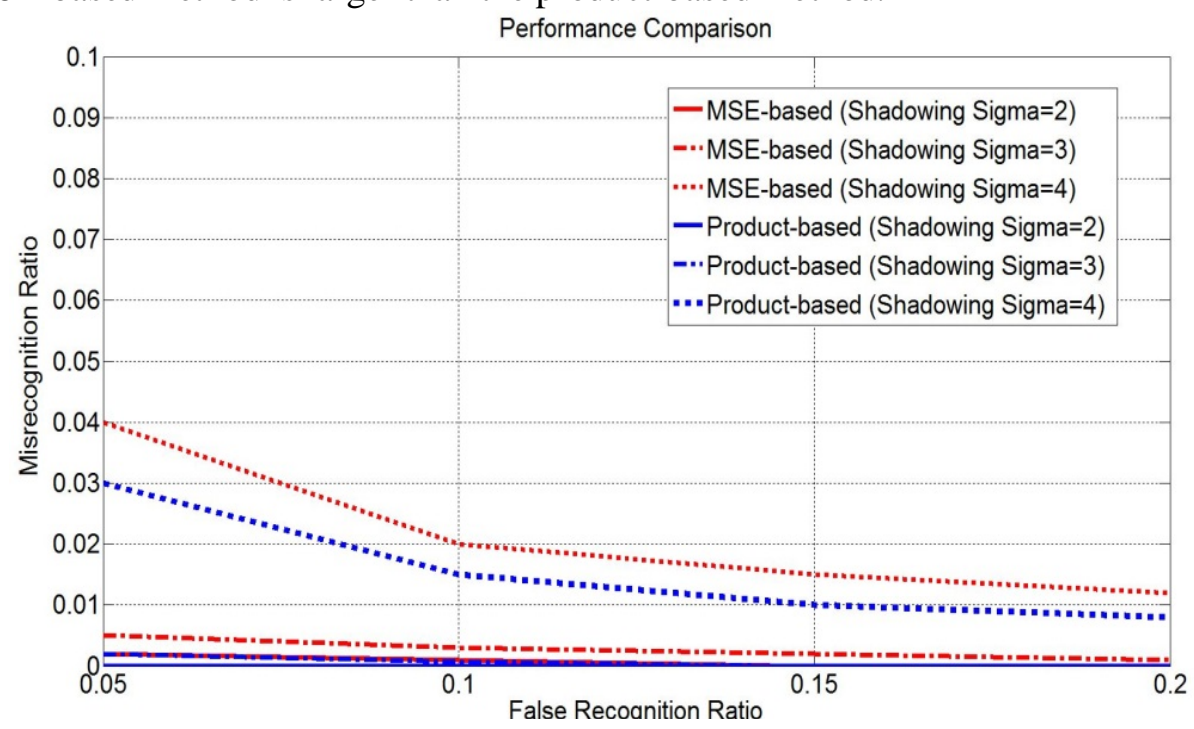

Fig. 20. Misrecognition ratio vs. false-recognition ratio for both the product-based and MSE-based methods. 


\section{Conclusions}

In this paper, we proposed a fast and reliable secondary system initialization protocol for cluster-based CR ad-hoc networks. The activation signal sequence sent by a cluster head is specially designed to have a specific frequency-time correlation characteristic. Therefore, at the member nodes, the system activation request channels are easily detected with FFT and autocorrelation operations. Because all SA transmission channels can be analyzed at the same time by the cluster's member nodes, the system setup time of the proposed method is not sensitive to the number of system activation channels of the $\mathrm{CH}$. In this paper, we also presented a reliable member node join and a common channel decision broadcasting mechanism. Compared with the conventional cyclic listening initialization method, the cluster joining delay is significantly reduced. Furthermore, SA channel detection using FFT and correlation operations can remain strong against the wireless channel variation so that the false recognition and misrecognition ratios can be maintained at very low bounds. The proposed channel initialization protocol uses a single-channel environment, and in the future, we intend to use a multi-channel environment.

\section{References}

[1] Wang, Beibei, and K. J. Liu., "Advances in cognitive radio networks: a survey," IEEE Journal of Selected Topics in Signal Processing, vol.5, no. 1, pp. 5-23, 2011. Article (CrossRef Link)

[2] Lingjie Duan, Lin Gao, Jianwei Huang, "Cooperative spectrum sharing: a contract-based approach,” IEEE Transactions on Mobile Computing, vol.13, no.1, pp. 174-187, 2014. Article (CrossRef Link)

[3] Jorswieck E.A., Badia L., Fahldieck T., Karipidis E., Jian Luo, "Spectrum sharing improves the network efficiency for cellular operators," IEEE Communicatuons Magazine, vol.52, no.3, pp. 129-136, 2014. Article (CrossRef Link)

[4] Chatzikokolakis K., Spapis P., Kaloxylos A., Alonistioti N., "Toward spectrum sharing: opportunities and technical enablers," IEEE Communicatuons Magazine, vol.53, no.7, pp. 26-33, 2015. Article (CrossRef Link)

[5] J. Mitola and G. Q. Maguire, "Cognitive radio: making software radios more personal," IEEE Personal Communications, vol. 6, no. 4, pp. 13-18, 1999. Article (CrossRef Link)

[6] Brandon F. Lo, "A survey of common control channel design in cognitive radio networks," Phys. Commun. (PHYCOM), vol. 4, no. 1, pp. 26-39, Mar. 2011. Article (CrossRef Link)

[7] C. Cormio and K. R. Chowdhury, "An adaptive multiple rendezvous control channel for cognitive radio wireless ad hoc networks," in Proc. of IEEE PERCOM Workshops, pp. 346-351, 2010. Article (CrossRef Link)

[8] DaSilva, Luiz, and Igor Guerreiro, "Sequence-based rendezvous for dynamic spectrum access," in Proc. of 3rd IEEE Symposium on New Frontiers in Dynamic Spectrum Access Networks (DySPAN 2008), pp. 1-7, 2008. Article (CrossRef Link)

[9] T. Chen, H. Zhang, G. M. Maggio, and I. Chlamtac, "CogMesh: a clusterbased cognitive radio network," in Proc. of 2nd IEEE Symposium on New Frontiers in Dynamic Spectrum Access Networks (DySPAN 2007), pp. 168-178, 2007. Article (CrossRef Link)

[10] Mi-Ryeong Kim, Sang-Jo Yoo, "Distributed coordination protocol for ad-hoc cognitive radio networks,” Journal of Communications and Networks, vol.14, no.1, pp. 51-62. 2012. Article (CrossRef Link)

[11] I-Hsun Chuang, Hsiao-Yun Wu, Yau-Hwang Kuo, "A fast blind rendezvous method by alternate hop-and-wait channel hopping in cognitive radio networks," IEEE Transactions on Mobile Computing, vol. 13, no. 10, pp. 2171 - 2184, 2014. Article (CrossRef Link) 
[12] Min-Gyu Kim, Sang-Jo Yoo, Young-Up Jang, Kwang-Eog Lee, "Reliable cluster-based common channel setup method for DSA CR networks," in Proc of fifth International Conference on Ubiquitous and Future Networks (ICUFN2013), pp. 155-160, 2013. Article (CrossRef Link)

[13] Sang-Jo Yoo, Ju-Tae Jang, Myunghwan Seo, Hyung-Weon Cho, "Common channel initialization protocol in cognitive radio ad-hoc networks," in Proc. of Seventh International Conference on Ubiquitous and Future Networks (ICUFN2015), pp. 754 759, 2015. Article (CrossRef Link)

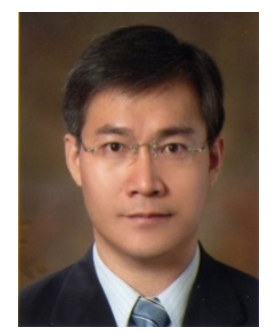

Sang-Jo Yoo received the B.S. degree in electronic communication engineering from Hanyang University, Seoul, Korea, in 1988 and the M.S. and Ph.D. degrees in electrical engineering from Korea Advanced Institute of Science and Technology (KAIST), in 1990 and in 2000, respectively. From 1990 to 2001, he was a member of technical staff at Korea Telecom Research and Development Group, where he worked in communication protocol conformance testing and network design fields. From September 1994 to August 1995 and from January 2007 to January 2008, he was a guest researcher at National Institute Standards and Technology (NIST), USA. Since 2001, he has been with Inha University, where he is currently a professor at Information and Communication Engineering Department. His current research interests include cognitive radio network protocols, seamless network mobility control, wireless network QoS, and wireless sensor networks.

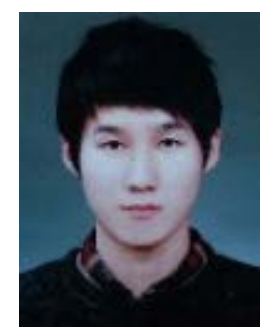

Ju-Tae Jang is expected to receive the B.S. degree in information and communication engineering from Inha University, Incheon, Korea in 2016. He joined the Multimedia Network Laboratory, Inha University, Incheon, Korea, led by Prof. S.J.Yoo, where he worked as a research student. His research interests include cognitive radio network and wireless communication.

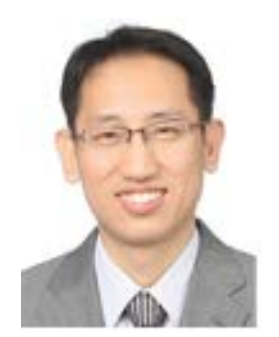

Myunghwan Seo received the B.S. degree in Department of Information Communications Engineering from Chungnam University, Korea, in 2001 and the M.S. and Ph.D. degrees in electrical engineering from Korea Advanced Institute of Science and Technology (KAIST), in 2004 and in 2009, respectively. Since 2009, he has been with Hanwha Tales. His current research interests include mobile ad-hoc network, wireless mesh network, network synchronization and military wireless communications.

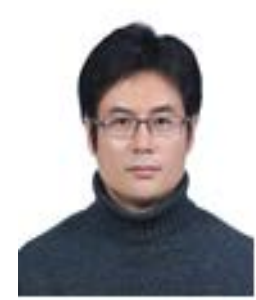

Hyung-Weon Cho received the B.S. and M.S. degrees in Electronic Engineering from Kwangwoon University, Korea, in 1992 and in 1994, respectively. He received Ph.D. degrees in electrical engineering from Yonsei University in 2012. Since 1997, he has been with Hanwha Tales. His current research interests include network design and optimization, communication systems and military wireless communications. 